\title{
Influencia de la regulación del trabajo del alumnado universitario en la implicación hacia las tareas
}

\author{
David HORTIGÜELA* y Ángel PÉREZ-PUEYO** \\ *Universidad de Burgos, **Universidad de León, España \\ (Received on September 16, 2014; Accepted on June 15, 2015)
}

RESUMEN: La presente investigación analiza la percepción que tiene el alumnado universitario sobre el proceso metodológico y evaluativo llevado a cabo en dos asignaturas, focalizándose en los factores de implicación hacia el trabajo y la regulación de las tareas. Participaron 241 alumnos de tres asignaturas del Grado en Primaria e Infantil de la Universidad de Burgos. Cada una de las asignaturas se dividió en dos grupos (A y B), cada uno impartido por un profesor mediante un enfoque pedagógico diferente. Se empleó una metodología de trabajo mixta mediante el diseño pretest-postest. Por un lado, cuantitativa, realizándose un análisis tanto descriptivo (medias y desviación típica por factores) como inferencial (ANOVAS). Por otro lado, cualitativa, realizando entrevistas a los dos docentes al finalizar el proceso. Se empleó un cuestionario validado de formación inicial del profesorado. Al finalizar la asignatura, los estudiantes del grupo A (registraron su trabajo de manera activa) manifestaron una mayor implicación hacia el trabajo, tanto en relación al pretest como respecto al grupo B (no regularon su trabajo). Dentro del grupo A fueron los alumnos con más titulaciones universitarias los que valoraron más positivamente la experiencia, mientras que en el grupo B las diferencias se encontraron en la variable independiente de la experiencia previa en el registro de trabajo. Los docentes otorgaron una importancia dispar al rol que desempeña la metodología en el aula.

Palabras clave: metodología mixta; regulación del trabajo; implicación en las tareas; percepción del alumnado; percepción del profesorado.

\section{The influence of regulating university student's workload on their attitude towards homework}

\begin{abstract}
This research analyzes the university students perception about the methodological and evaluative process conducted in two subjects. It affects factors involved to work and regulation of wokload. 241 students from three subjects Degree in Primary and Infant University of Burgos participated. Each of the subjects were divided into two groups (A and B), each taught by a teacher through different pedagogical approach. A mixed methodology was used by pretest-posttest design. On one hand, quantitative, through a descriptive analysis (mean and standard deviation factors) and inferential (ANOVA). On the other hand, qualitative, through an interview with the two teachers to complete the process. A validated questionnaire initial teacher was employed. At the end of the course, students in group A (regulated their work actively) reported greater involvement to work, both in relation to pretest as compared to group B (not regulated their work). Within group A were students with more university degrees who value more positive experience. In group B were differences in the independent variable of previous experience in the recorded work actively. Teachers gave a different importance to the role played by the methodology in the classroom.
\end{abstract}

Keywords: Mixed methodology; regulation workload; involvement in tasks; student perception; teachers perception.

Correspondencia: David Hortigüela Alcalá. Facultad de Educación. Calle Villadiego s/n. 09001. Burgos.dhortiguela@ubu.es. 


\section{Introducción}

En la actualidad cada vez más el sistema universitario ha de replantearse cuáles son las principales finalidades en cuanto a la formación del estudiante, y como consecuencia, qué modelos metodológicos son los más idóneos para que verdaderamente se genere un aprendizaje sólido, útil y transferible (Powell y Robson, 2014). Como indican Ballesta, Izquierdo y Romero (2011), antes de atender únicamente a modelos macro estructurales que arrojen cifras cuantitativas acerca de la relación entre universidad, empleabilidad y aspectos socioeconómicos, es de suma importancia reflexionar sobre la manera en la que se desarrollan competencias en cada una de las materias, valorando para ello la percepción del alumnado sobre lo aprendido. Independientemente de las características intrínsecas de cada titulación, especialidad o materia, el enfoque metodológico de la enseñanza debe centrarse en el aprendizaje como pilar básico del proceso, siendo necesario por lo tanto la delimitación de estrategias concretas de actuación que posibiliten su mayor implicación a través de la autoevaluación de su trabajo (MacDonald, 2012). Esta idea, aunque puede parecer que ya está instaurada y consolidada en el ámbito universitario, algunas experiencias como las de Vavpotic, Zvanut y Trobec (2013) reflejan que son los modelos tradicionales los que siguen imperando en el aula, centrados fundamentalmente en la impartición de contenidos por parte del profesor y quedando relegada la función del estudiante a la recogida y memorización de la información. Estos modelos metodológicos son valorados por el alumno como menos motivantes y útiles en otros contextos (Hortigüela, Pérez Pueyo y Abella, 2015).

Por lo tanto, sigue siendo trascendental el rol que tiene la formación permanente del profesorado en la construcción de pautas y protocolos de actuación en el aula, articulados éstos a partir de modelos de carácter innovador (Van Looy y Goegebeur, 2007). No se trata de que el docente modifique radicalmente la forma de concebir sus prácticas educativas, sino de reflexionar sobre el calado que tienen en el aprendizaje que se pretende que construya el alumnado. Para ello es fundamental trabajar conjuntamente, convirtiéndose los ciclos de investigación-acción en una herramienta de verdadero interés tanto para obtener un feedback real del alumnado como para solventar aquellas problemáticas que pueden generarse en el día a día (Navarro, Campos y Uceda, 2014). Si queremos que el alumno se implique en aquello que aprende es necesario generar autonomía hacia el aprendizaje, y para ello es preciso delimitar desde el comienzo aquello que se va a enseñar y los criterios utilizados para evaluarlo (Picón, 2012).

En consonancia con las demandas establecidas con el Espacio Europeo de Educación Superior (EEES), y bajo la principal premisa de que el alumno sea capaz de interiorizar el aprendizaje y poder usar el mismo en su cotidianeidad, ya sea esta profesional o personal,la metodología empleada en el aula ha de favorecer la regulación del trabajo por parte del estudiante. Experiencias en esta línea demuestran que la excesiva dependencia del estudiante hacia el profesor sobre los contenidos impartidos y el tipo de trabajos demandados limita las verdaderas opciones de aprendizaje (Jones, 2014). En relación a esto, diferentes estrategias aplicadas en evaluación formativa, tanto en el ámbito nacional como internacional, muestran resultados favorables sobre la construcción de aprendizajes por parte del estudiante cuando éste trabaja de manera autorregulada (Fraile, López-Pastor, Castejón y Romero, 2013). Esta forma de proceder se asocia, cada vez más, al empleo de las nuevas tecnologías (NNTT), existiendo 
una gran cantidad de instrumentos y estrategias que permiten tanto una profundización en el conocimiento como una exploración en nuevos campos del saber. Experiencias como las de Sosa, López y Díaz (2014) demuestran cómo el uso de plataformas virtuales a través de la elaboración de masive open online course (MOOC) mejoran la implicación del alumno hacia las tareas demandadas, realizando más aportaciones y de manera más constructiva. Modelos pedagógicos como el flipped classroom facilitan estos procesos y garantizan un empleo más eficaz del tiempo de clase mediante el trabajo en grupo (Vaughan, 2014).

Queda por tanto de manifiesto la importancia que tiene el tipo de metodología empleada en la percepción del estudiante sobre su motivación y el aprendizaje construido, por lo que parece trascendental reflexionar sobre el uso de las estrategias docentes utilizadas. Esto se asocia directamente con la adquisición de futuras competencias profesionales, independientemente de la titulación en la que se enmarque el proceso. Parece lógico otorgar importancia a los fines que configuran la enseñanza en cada una de las asignaturas o titulaciones, adecuando y adaptando las guías docentes aúna sociedad tan cambiante como en la que nos encontramos (Varbelow, 2014). Esto conlleva una actualización en la formación permanente del profesorado universitario, vinculando lo que se enseña en las aulas y lo que se demanda fuera de las mimas (Guzmán, 2012).

Esta valoración que realiza el estudiante sobre la metodología vivenciada depende directamente de las variables independientes implicadas. A modo de ejemplo, dentro de las titulaciones de maestro de infantil y de primaria, estrategias fundamentadas en el desarrollo de sesiones prácticas en contextos escolares son valoradas por el alumnado como verdaderamente útiles en contextos profesionales futuros (Newberry, 2013). Es fundamental adaptar las estrategias a las características de la clase, la titulación en la que se imparte docencia y/o las experiencias y conocimientos previos del alumno, ya que las pretensiones de los estudiantes y los fines de los aprendizajes serán diferentes.

Por lo tanto, en esta investigación, y debido a la importancia que tienen las estrategias de enseñanza, se analizará la percepción del alumno sobre el papel que tiene la metodología recibida, incidiendo en cómo la variable independiente de la regulación del trabajo influye en su implicación hacia las tareas. Para ello se han utilizado dos enfoques de enseñanza distintos. Esto permite arrojar nuevos y aplicables datos sobre la problemática estudiada.

\section{Objetivos}

- Comparar la percepción que tiene el alumnado sobre la implicación en las tareas a partir de la regulación del trabajo implícita en la metodología utilizada en cada uno de los grupos.

- Analizar la influencia que tienen las variables de edad, experiencia previa en el registro de trabajo y posesión de titulaciones universitarias en la valoración positiva de la asignatura para cada uno de los grupos.

- Contrastar la percepción que tienen los docentes que han impartido las asignaturas sobre el rol que desempeña la metodología para conseguir mayor implicación y gestión del trabajo. 


\section{Método}

\section{Participantes}

En esta investigación participan 241 alumnos de tres asignaturas del grado de Primaria e Infantil de la Universidad de Burgos. 157 (65,2 \%) eran alumnas y 84 alumnos (34,8\%). La media de edad era de 21.39 años (DT = 2.52). Cada una de las asignaturas se divide en dos grupos, A y B, siendo impartida cada uno con una metodología diferente. Estos criterios de reparto atienden a la designación que se hace en la secretaría de la Facultad a partir del número de alumnos matriculados. Los alumnos que han participado en el grupo A (horario de mañana) son 129 y los que lo han hecho en el grupo B (horario de tarde) son 112.

Tras realizar la prueba de SHAPIRO-WILK se observa que la muestra responde a parámetros de normalidad $(p=.141)$. El profesor del grupo A tiene cinco años de experiencia en el ámbito universitario mientras que el del B 28, y ambos siempre han trabajado en la Universidad de Burgos. El docente del grupo A tiene una mayor formación académica, así como un mayor número de contribuciones al ámbito académico y científico (congresos, publicaciones en revistas, libros...). El del grupo B ha impartido más años la asignatura, así como otras de psicomotricidad y actividad física orientadas a un carácter rehabilitador. Las tres asignaturas del estudio tienen asignada carga docente al Área de Didáctica de la Expresión Corporal, de la cual forman parte los dos docentes.

En la Tabla I se puede observar el número de casos estudiados, las asignaturas, los grados a los que pertenecen, el curso y los participantes de cada uno de los grupos.

Tabla 1. Participantes en el estudio (caso, asignatura, grado y número de participantes para cada una de las vías.

\begin{tabular}{clcccc}
\hline CASO & ASIGNAURA & GRADO & CURSO & \multicolumn{2}{c}{ GRUPO/N ${ }^{\text {PARTICIPANTES }}$} \\
\hline \multirow{2}{*}{1} & $\begin{array}{l}\text { Educación Física } \\
\text { y su Didáctica }\end{array}$ & Primaria & $2^{\circ}$ & Grupo A/54 & Grupo B/ 48 \\
\hline 2 & Juego Educativo & Primaria & $4^{\circ}$ & Grupo A/ 32 & Grupo B/ 29 \\
\hline \multirow{2}{*}{3} & $\begin{array}{l}\text { Desarrollo Psico. } \\
\text { I }\end{array}$ & Infantil & $3^{\circ}$ & Grupo A/ 43 & Grupo B/ 35 \\
\hline & & TOTAL & 129 & 112 \\
\hline
\end{tabular}

\section{Instrumentos}

\section{Cuantitativos}

Se ha utilizado el cuestionario validado de evaluación sobre la formación inicial del profesorado (Castejón, Santos y Palacios, 2015). El proceso de construcción y validación del mismo se realizó a partir de la formulación de los objetivos y sus variables de estudio, la especificación de su contenido y la reformulación de sus ítems hasta alcanzar su convergencia.

Se obtiene una fiabilidad del instrumento según el alfa de Cronbach de .835, superior al límite inferior que según Corbetta (2007) es aceptado como fiable. Respecto a la estabilidad temporal de los datos y a partir de la realización de la correlación de Pearson se obtiene una r de 0.88 . La validez del cuestionario queda patente al comprobar cómo en la prueba piloto se 
confirma el significado previsto de las variables que se pretenden medir, reflejando la utilidad práctica del instrumento. A partir de la identificación de las variables predictivas (aquello que se quiere medir) del cuestionario se definieron operacionalmente cada uno de los ítems por los que está compuesto, relacionados estrechamente con los objetivos de la presente investigación.

Para el cuestionario final se realizó un Análisis Factorial Exploratorio mediante el método de extracción de Componentes Principales, donde se quiere ver la idoneidad de los datos con el fin de evaluar la bondad del ajuste de los datos. Se obtienen valores adecuados para el índice KMO de 0.834 como en el test de esfericidad de Barlett ( $>$ > .00). Este test presenta un valor de Chi-cuadrado de 2312.212 y 32 grados de libertad. Se realiza un análisis factorial confirmatorio con el fin de extraer los factores de estudio a partir de los ítems que integran el cuestionario. Los índices obtenidos en la matriz de covarianzas, presentaron ajustes satisfactorios para el índice RMSEA (Root Mean Square Error Aproximation) $=0.062$. En este índice los valores inferiores a .05 indican un buen ajuste, y valores de hasta .08 representan errores razonables de aproximación (Herrero, 2010).

En el CFI (Comparative Fit Index) y GFI (Goodness of Fit Index), se alcanza un valor de 0.91, y 0.93, indicativo de buen ajuste.

Esta escala tiene un total de 20 cuestiones a las que los estudiantes responden en grado al acuerdo con el enunciado en una escala tipo Likert, cuyos valores van desde 1 (Nada) hasta 5 (Mucho).

El análisis factorial realizado en la validación del cuestionario muestra dos factores que integran el mismo:

1- $\quad$ Implicación hacia las tareas (11 ítems): se abordan ítems relacionados con la predisposición con la que el alumno afronta las tareas, su actitud hacia la resolución de las mismas y las estrategias utilizadas para profundizar en los conocimientos adquiridos.

2- $\quad$ Regulación del trabajo (9 ítems): este factor integra cuestiones vinculadas con la forma en la que el estudiante organiza el trabajo, la delimitación de roles y responsabilidades que se establecen en los grupos de trabajo y las herramientas empleadas para registrar los avances encontrados.

\section{Cualitativos}

Se emplea una entrevista semi-estructurada a los docentes al finalizar la asignatura con el fin de profundizar en los datos obtenidos. En función de un guion abierto se plantearon unas cuestiones para explorar los pensamientos del docente (Patton, 2002). Como la naturaleza de la entrevista era semi-estructurada, los investigadores pudieron añadir nuevas preguntas en base a las respuestas de los entrevistados para convertir la entrevista en una conversación. Este formato abierto permite explorar nuevas áreas para producir datos más ricos (Smith y Osborn, 2003). Son cuatro cuestiones principales las que se plantean a los dos profesores al finalizar la asignatura en relación a los factores de estudio; 1 )- ¿Consideras que la metodología utilizada en el aula repercute en la motivación del alumno hacia lo que se enseña?, 2)- ¿Cuál es la mejor manera para que el alumno se implique en las tareas que le demandamos? 3)- ¿Crees conveniente facilitar estrategias que permitan al alumno regular su trabajo? 4)- ¿Te parece relevante que el alumno pueda implicarse en su evaluación? ¿Cómo puede conseguirse?

A partir de toda la información obtenida se generaron tres categorías de análisis: 1“Importancia del rol de la metodología y la evaluación”, 2- “Estrategias para fomentar la 
implicación del alumno en las tareas", 3- "Proceso de autorregulación del proceso de enseñanza”.

\section{Diseño y procedimiento}

Todos los estudiantes de las tres asignaturas han cumplimentado individualmente el cuestionario al finalizar el proceso. En todo momento se garantizó el anonimato para que las respuestas de los estudiantes fueran lo más sinceras posibles, al igual que se garantizó la confidencialidad en el tratamiento de los datos.

Por tanto, y a pesar de que los objetivos a conseguir en las asignaturas para cada uno de los grupos son los mismos, la metodología empleada por cada profesor varía sustancialmente:

- Grupo A: se ha trabajado en grupos, aplicando un sistema de evaluación formativa mediante el feedback establecido por el profesor para la mejora del trabajo. Cada uno de los grupos registra su trabajo semanalmente, indicando el número de horas invertidas en cada procedimiento de evaluación. Esta autorregulación del trabajo es revisado por el docente periódicamente. Además cada grupo realiza coevaluaciones intragrupales, valorando a cada compañero de manera consensuada. Estas coevaluaciones y autoevaluaciones de grupo permiten tanto un mayor control del trabajo realizado como un incremento en la responsabilidad individual de los integrantes.

Grupo B: no se aplica un sistema de evaluación formativa ni se articulan procedimientos consensuados para que el alumno registre su trabajo a lo largo de la asignatura. Se trabaja en grupo, pero no existe una sistematización sobre las valoraciones intragrupales y la percepción del trabajo realizado. No se articulan procedimientos evaluativos que permitan a los integrantes de grupos ser conscientes y consecuentes del trabajo realizado y de la mejora del mismo.

\section{Análisis empleado}

La metodología de la investigación ha sido mixta, empleándose tanto un análisis cuantitativo (descriptivo e inferencial) como cualitativo (entrevistas a docentes). Se emplea un pretest-postest, analizando en qué medida el tipo de metodología utilizada influye en la implicación hacia las tareas y la regulación del trabajo del alumno. La parte cualitativa del estudio se centra en el análisis de la valoración que hacen los docentes de cada grupo sobre la relevancia que tiene el uso de las estrategias metodológicas empleadas, lo que favorece el análisis de las percepciones de los alumnos. Esta complementariedad en el tratamiento de los datos otorgará un visionado más global de los resultados obtenidos, así como una mayor comprensión de los mismos. Además favorece la transformación de los procesos educativos desarrollados (Hall y Ryan, 2011).

\section{Cuantitativo}

Por un lado es descriptivo (medias) y por otro inferencial (ANOVAS), basándose en los factores de análisis obtenidos en la investigación a partir de los objetivos del estudio. Mediante el pretest-postest en cada uno de los grupos se comprueba cómo varía la percepción del alumno en relación a las variables independientes analizadas. Para analizar los datos se utiliza el paquete estadístico SPSS. 


\section{Cualitativo}

Se obtienen los datos a partir de la información recogida en las entrevistas realizadas a los docentes. La información extraída se analiza a través de la exploración en la temática del contenido (Libarkin y Kurdziel, 2002) y la comparación constante entre los datos (Denzin y Lincoln, 1994). Se codifican los extractos de textos coincidentes con los patrones cruzados mediante la triangulación y saturación (Saldaña, 2009). La confiabilidad fue apoyada a través de la retroalimentación continua y el análisis participativo por parte de los investigadores que revisaron y perfeccionaron las categorías emergentes, para que los resultados pudieran ser considerados confiables, creíbles y transferibles (Lincoln y Guba, 1985). El objetivo era utilizar la información obtenida para dotarla de una mayor comprensibilidad a través de la transferencia de los resultados.

Para recapitular, organizar y obtener la saturación de los datos a partir de las categorías generadas en las preguntas realizadas a los dos docentes se ha utilizado el programa de computación WEFT QDA. El acrónimo empleado para el docente que utilizó el registro del trabajo fue DRT (docente registro trabajo) y para el que no lo utilizó fue DNR (docente no registro).

Las categorías utilizadas se generan a partir de los objetivos del estudio. Los procedimientos de análisis que se han empleado se ciñen a las propuestas de fragmentación y articulación de la «GroundedTheory» (Strauss \&Corbin, 2002). Se realizó una codificación abierta, con categorías emergentes; codificación axial, en torno a la agrupación de las categorías emergentes en familias de subcategorías; y codificación selectiva, mediante un trabajo específico que condujo a la elaboración de un modelo interpretativo. En cada una de las entrevistas se identifica cada extracto de texto con una de las categorías delimitadas, lo que favorece la obtención de la saturación de la información a partir de la sección, recopilación y contabilización de cada uno de los fragmentos.

Análisis descriptivos

\section{Resultados}

Tabla 2. Comparación de medias por factores para cada uno de los grupos en el pretest-postest (nivel de significación en las diferencias: *p <.05)

\begin{tabular}{lccccccc}
\hline & \multicolumn{3}{c}{ PRE-TEST } & \multicolumn{3}{c}{ POST-TEST } \\
\hline & $\mathbf{N}$ & Media & DT & Var. & Media & DT & Var. \\
\hline & \multicolumn{6}{c}{ Grupo registro trabajo (a) } \\
\hline F.1. Implicación hacia las tareas & 129 & 3.36 & .213 & .045 & $4.29^{\text {aa }}$ & .123 & .015 \\
\hline F.2. Regulación del trabajo & 129 & 3.56 & .136 & .018 & 4.12 & .187 & .034 \\
\hline & \multicolumn{6}{c}{ Grupo no registro trabajo (b) } \\
\hline F.1. Implicación hacia las tareas & 112 & 3.41 & .241 & .058 & $3.27^{\text {ba }}$ & .241 & .058 \\
\hline F.2. Regulación del trabajo & 112 & 3.27 & .291 & .084 & 3.41 & .311 & .096 \\
\hline
\end{tabular}

Nota: Superíndices distintos entre grupos señalan diferencias significativas a nivel .05

Se refleja cómo se obtienen diferencias significativas en el factor 1 entre grupos en el postest, siendo el grupo A el que obtiene la media más elevada. Estas diferencias también se encuentran en este grupo y factor entre el pretest y postest. En el pretest existe una homogeneidad en los valores de las medias entre los dos grupos, no llegando en ningún caso a 
cuatro puntos. En el postest las medias son más elevadas en los dos factores en el grupo que registro estructuradamente el trabajo.

\section{Análisis inferencial: ANOVAS}

Se realizó un análisis Bonferroni y Post Hoc en relación a los ítems relacionados con la valoración positiva de la asignatura realizada por el alumno, generándose una variable de escala para su contraste. Esta variable dependiente se relaciona con otras independientes. La primera de ellas es la edad, dividida en 1- "menos de 21", 2- “entre 21 y 23” y 3- "más de 23". La segunda es la experiencia previa en el registro de trabajo; 1- "ninguna vez", 2- "una vez", y 3- "más de una vez". La tercera es las titulaciones universitarias previas del alumno; 1"ninguna”, 2- “una” y 3- "más de una” (ver tabla 3).

Tabla 3. Resumen de ANOVA (Bonferroni) para cada una de las variables independientes analizadas en el postest (edad, experiencia previa en el registro de trabajo y titulaciones universitarias previas).

\begin{tabular}{cccc}
\hline VALORACIÓN POSITIVA DE LA ASIGNATURA & $F$ & $g l$ & $p$ \\
\hline Gruporegistro de trabajo (a) & & & \\
\hline Edad & 96.71 & 1 & .135 \\
\hline Experiencia previa en registro de trabajo & 91.22 & 2 & .223 \\
\hline Titulaciones universitarias previas & 73.45 & 1 & $\mathbf{. 0 1 2}^{*}$ \\
\hline Grupos no registro de trabajo (b) & & & \\
\hline Edad & 98.41 & 1 & .131 \\
\hline Experiencia previa en registro de trabajo & 91.13 & 2 & $\mathbf{. 0 0 6} * *$ \\
\hline Titulaciones universitarias previas & 84.31 & 1 & .213 \\
\hline
\end{tabular}

${ }^{*} \mathrm{p}<.05$ entre “ninguna” (media 3.63) y “más de una” (media 4.71)

** $\mathrm{p}<.05$ entre “más de una vez" (media 3.03) y “ninguna vez” (media 3.89)

En el grupo que registró el trabajo las diferencias se encuentran en las titulaciones universitarias previas que tiene el alumno $\left(\mathrm{F}_{(129)}=73.45, \mathrm{p}<.012\right)$, siendo los que tenían más de una los que más valoran la experiencia recibida. En el grupo B las diferencias se alcanzan en la variable de la experiencia en el registro del trabajo de manera estructurada en otras asignaturas $\left(\mathrm{F}_{(112)}=91.13, \mathrm{p}<.006\right)$. Aquí los que lo habían vivenciado más de una vez valoraron menos positivamente la asignatura.

\section{Análisis cualitativo}

Mediante el análisis de patrones cruzados se presentan el número de extractos literales de texto resultantes en cada categoría. Como el tipo de entrevista realizada es de carácter semiabierto se llevó a cabo una retroalimentación a lo largo de la misma con cada docente para profundizar en cada una de las respuestas. Toda la información recabada se seleccionó y asignó a las tres categorías, vinculadas directamente con los objetivos de estudio.

1- $\quad$ Importancia del rol de la metodología y la evaluación (153 extractos de texto). El profesor del grupo A otorga una mayor importancia al tipo de metodología empleada, considerando que el rol que desempeñe el alumno en la asignatura depende fundamentalmente de las estrategias utilizadas por el docente, algo que no considera el profesor del grupo B: 
"La metodología es la clave del proceso de enseñanza [...]" "Hay que definir procedimientos que hagan atractivo el aprendizaje al alumno" (DRT). "Cada profesor tiene su metodología, mejor o peor, al final lo que importa es que el alumno aprenda lo que corresponda” (DNR).

La formación académica y la experiencia son factores que determinan esta concepción y valoración sobre la metodología empleada:

"Actualmente hay una gran cantidad de estudios y propuestas que demuestran la importancia que tiene el tipo de metodología empleada [...]" "Cualquier congreso o actividad formativa en el ámbito de la docencia va dirigida en este sentido" (DRT). "Al final esto va por modas [...]" "Hace 30 años se hablaba de lo mismo pero de otra manera. El alumno tiene que aprender lo que tiene que aprender y eso no cambia por unas u otras influencias educativas" (DNR).

2- $\quad$ Estrategias para fomentar la implicación del alumno en las tareas (161 extractos de texto). El profesor del grupo $\mathrm{B}$, al contrario que el del $\mathrm{A}$, no cree que en estudios universitarios haya que dar especial transcendencia a este aspecto:

"En estudios no obligatorios como la universidad la motivación debería estar presente [...]" "Si un alumno elige es porque tiene que implicarse" (DNR). "Independientemente de lo que se enseñe han de buscarse estrategias que favorezcan la implicación del alumno en aquello que aprende, si no estaremos cayendo en modelos reproductivos de información" (DRT).

Además, el docente del grupo A otorga una importancia más elevada a este hecho cuando se trabaja en titulaciones relacionadas con la formación inicial del profesorado, algo que no sucede con el grupo B:

"Conseguir que el alumno se implique es fundamental, pero mucho más cuando estamos enseñando a futuros maestros [...]" "Es una titulación altamente vocacional y la responsabilidad en la formación es elevadísima, ya que está en juego el futuro de la sociedad" (DRT). "Todo alumno universitario ha de implicarse (por su bien) hacia aquello que aprende [...]" "Un arquitecto construirá edificios, un profesor enseñará a niños, un cirujano salvará vidas [...]" "Pero que no se nos olvide que nosotros enseñamos contenidos, no formas de motivarse hacia lo que se aprende" (DNR).

3- $\quad$ Proceso de autorregulación del proceso de enseñanza (183 extractos de texto). El profesor del grupo A lo considera trascendental, siendo el docente un agente activo dentro de este proceso. El del grupo B expresa que la autonomía a esta edad ha de ser ya elevada, por lo que no hay que invertir tiempo en esto:

"Es fundamental que el alumno registre y sea consciente del trabajo realizado y el que le queda por hacer, estableciendo el docente el feedback adecuado" (DRT). "Yo bastante tengo con enseñar todo lo que toca en tan poco tiempo, el alumno ha de saber regularse [...] (DNR).

Esta percepción de los docentes está claramente influenciada por las experiencias previas que han tenido cuando eran estudiantes:

"Cuando yo era estudiante cada uno se autorregulaba la manera de estudiar como consideraba, y la verdad es que los resultados no eran nada malos [...]" "Sinceramente creo 
los antiguos maestros especialistas salían mejor formados que con las actuales menciones, a pesar de que todo ahora parezca mucho más innovador" (DNR). "Personalmente he vivenciado procesos formativos en los que se posibilitaba que el alumno fuera miembro activo en la regulación de su trabajo y también he sufrido otros en los que los docentes se limitaban a exponer información [...]" "Las diferencias de aprendizaje son abismales [...]" (DRT).

\section{Discusión}

Se ha observado cómo la metodología empleada por el docente en el ámbito universitario, en concreto, prácticas asociadas a la regulación del trabajo de manera autónoma y a la coevaluación y autoevaluación a lo largo del proceso, han repercutido favorablemente en la implicación del alumno hacia el aprendizaje. Este factor es el que ha experimentado cambios significativos en el postest, favorables hacia el grupo que llevó a cabo la metodología abierta y participativa. Algunas experiencias (Hortigüela, Pérez Pueyo, y Abella, 2015) determinan la relevancia que tiene valorar de manera intencional la percepción del alumnado sobre su proceso de aprendizaje, ya que favorece una comprensión más profunda sobre cómo ha de estructurarse y plantearse la asignatura. Esto sin duda conllevará un replanteamiento de la metodología utilizada por el docente, repercutiendo en la adecuación de las estrategias, materiales e instrumentos a utilizar, así como en la incidencia en la transferencia de los aprendizajes (Aguaded y Moraleda, 2015). Es por ello que en la formación inicial del profesorado deben generarse procesos de enseñanza que permitan al futuro maestro tener herramientas para aplicar en el aula en un futuro cercano (Delgado y Casado, 2014). Esto ha de ser valorado por el profesorado, y como consecuencia adaptar las competencias de las asignaturas a las exigencias en los procedimientos de evaluación empleados.

Respecto a la valoración positiva del alumno sobre la asignatura cursada, dentro del grupo A fue la variable independiente de las titulaciones universitarias previas en la que se obtuvieron diferencias. Los alumnos con más de una titulación percibieron el proceso desarrollado como más positivo. Esto demuestra la relación positiva existente entre experiencias formativas del alumno y el empleo de metodologías que permitan al estudiante gestionar su proceso de enseñanza (Kanyal, 2014). Sin embargo, en el grupo B las diferencias se observan en la variable de las experiencias que los alumnos han tenido en el registro de horas y coevaluaciones y autoevaluaciones en otras asignaturas, siendo los que más han experimentado los que peor valoran la asignatura recibida. Esto determina que aquellos alumnos que han vivenciado experiencias en las que se les ha permitido ser autónomos en la regulación de su trabajo se hacen más críticos a la hora valorar procesos basados en metodologías más tradicionales y con menos capacidad de intervención y regulación (Covill, 2011). En la variable edad no se encuentran diferencias en ninguno de los grupos. Sin embargo otras experiencias educativas (Low, Polanin y Espelage, 2013) basadas en el uso de las NNTT indican una percepción más positiva entre el alumnado más joven.

La parte cualitativa del estudio ha reflejado la consonancia existente entre la percepción del alumnado y la metodología empleada por cada uno de los docentes. El grupo que recibió la metodología fundamentada en el registro del trabajo estructurado fue el que manifestó una percepción más positiva sobre el proceso de aprendizaje recibido. Relacionado con ello, el profesor que impartió este grupo otorga una mayor relevancia al rol que tiene las estrategias de 
enseñanza en el proceso educativo. Del mismo modo, las percepciones más bajas se encontraron en el grupo B, impartido por el docente que empleó una metodología de carácter más tradicional.

El docente del grupo A otorga una mayor importancia a la delimitación de estrategias que permitan al alumno implicarse en su proceso, regular su trabajo y fomentar su autonomía. Sin embargo el del grupo B no considera relevante las estrategias metodológicas empleadas, centrándose fundamentalmente en la impartición de contenidos de la asignatura. Experiencias como la de Vrieling, Bastiaens y Stijnen (2012) reflejan una linealidad entre la implicación y motivación que presenta el docente hacia las clases y aquel aprendizaje que manifiesta el alumno haber adquirido.

Consideramos que el presente estudio puede ser relevante para todos aquellos docentes universitarios interesados en la importancia que tiene la metodología aplicada en el aula y las repercusiones derivadas en la motivación y aprendizaje del alumno. También para maestros y profesores de etapas educativas obligatorias, debido a la cantidad de variables y posibilidades existentes a la hora de implicar al alumno en aquello que aprende. El artículo presenta algunas limitaciones. En primer lugar únicamente atiende a datos de una facultad, por lo que podría ampliarse la experiencia a otras titulaciones de la misma universidad o incluso de otras. Por otro lado podría ser interesante contrastar estos resultados con la percepción que tengan los alumnos egresados, comprobando la incidencia que tienen las experiencias vivenciadas en las asignaturas en la transferencia de los aprendizajes adquiridos a contextos profesionales reales.

El principal aporte de esta investigación es el análisis realizado sobre el contraste entre la percepción del docente y el discente a través del empleo de un método mixto. Se ha determinado cómo el uso de una evaluación formativa a través de la realización de feedbacks con el alumno, el asesoramiento en la realización de las tareas y el registro del trabajo de manera regular ha favorecido su implicación hacia las tareas. Además, se demuestra cómo la importancia que otorgue el docente a las estrategias de aula repercute directamente en la percepción del estudiante. Puede afirmarse por lo tanto que la metodología utilizada en clase es un factor relevante para la motivación del alumnado. Hemos de precisar que el concepto de metodología docente es muy amplio, ya que engloba diversidad de aspectos como la manera de abordar los contenidos, la forma de relacionarse con el alumnado y/o el tipo de agrupamientos y tareas demandadas. Por ello sería conveniente demostrar en trabajos futuros cuáles son las subdimensiones concretas de la metodología que más influyen en las percepciones del estudiante.

\section{Agradecimientos}

Este estudio se ha llevado a cabo dentro del proyecto de $\mathrm{I}+\mathrm{D}+\mathrm{i}$ : "La competencias docentes en la formación inicial del profesorado de educación física”. Convocatoria de noviembre de 2013 del Programa Estatal de Investigación, Desarrollo e Innovación Orientada a los Retos de la Sociedad, en el marco del Plan Estatal de Investigación Científica y Técnica y de Innovación 2013-2016. Referencia: EDU 2013-42024-R. Duración: 3 años (2014-2016). 


\section{Referencias}

Aguaded, M.C., y Moraleda, E. (2015). Opinión del alumnado acerca de los cambios metodológicos de enseñanza en la asignatura. Infancias Imágenes, 13, 61-69.

Ballesta, F.J., Izquierdo, T., y Romero, E. (2011). Percepción del alumnado de Pedagogía ante el uso de metodologías activas. Educatio siglo XXI: Revista de la Facultad de Educación, 29, 353-368.

Castejón, F. J., Santos, M., y Palacios, A. (2015). Cuestionario sobre metodología y evaluación en formación inicial en Educación Física. Revista Internacional de Medicina y Ciencias de la Actividad Física y el Deporte, 15 (58), 245-267.

Cohn, P. J. (1991). An exploratory study on peak performance in golf. The Sport Psychologist, $5,1-14$.

Corbetta, P. (2007). Metodologías y técnicas de investigación social. Madrid: McGrawHill.

Covill, A. (2011). College Students' Perceptions of the Traditional Lecture Method. College Student Journal, 45(1), 92-101.

Delgado, V., y Casado, R. (2014). Radiografía de la formación del profesorado en la Universidad de Burgos: evolución y análisis de planes y estrategias formativas. Bordón. Revista de Pedagogía, 66(2), 43-60.

Denzin, N. K., y Lincoln, Y. S. (1994). Handbook of Qualitative Research. Thousand Oaks, CA: Sage.

Fraile, A., López-Pastor, V. M., Castejón, F. J., y Romero, R. (2013). La evaluación formativa en docencia universitaria y el rendimiento académico del alumnado. Revista Aula Abierta, 41(2), 23-34.

Guzmán, J.R. (2012). Comunidades de aprendizaje y formación del profesorado. Tendencias Pedagógicas, 19, 67-86.

Hall, J., y Ryan, K. (2011). Educational Accountability: A Qualitatively Driven MixedMethods Approach. Qualitative Inquiry, 17(1), 105-115.

Herrero, J. (2010). El Análisis Factorial Confirmatorio en el estudio de la Estructura y Estabilidad de los Instrumentos de Evaluación: Un ejemplo con el Cuestionario de Autoestima CA-14. Intervención Psicosocial, 19(3), 289-300.

Hortigüela, D., Pérez Pueyo, A. y Abella, V. (2015). Perspectiva del alumnado sobre la evaluación tradicional y la evaluación formativa. Contraste de grupos en las mismas asignaturas. REICE. Revista Iberoamericana sobre Calidad, Eficacia y Cambio en Educación, 13(1), 35-48. Recuperado de http://www.rinace.net/reice/numeros/arts/vol13num1/art3.pdf.

Jones, S. (2014). Assessing the Science Knowledge of University Students: Perils, Pitfalls and Possibilities.Journal of Learning Design, 7(2), 16-27.

Kanyal, M. (2014).Early Childhood Studies--Students' Participation in the Development of a Learning Space in a Higher Education Institution. Management in Education, 28(4), 149-155.

Libarkin, J. C., yKurdziel. J. P. (2002). Research methodologies in science education: Qualitative data. Journal of Geoscience Education, 50, 195-200.

Lincoln, Y. S., yGuba, E. (1985). Naturalistic inquiry. Newbury Park, CA: Sage. 
Low, S., Polanin, J., yEspelage, D. (2013). The Role of Social Networks in Physical and Relational Aggression among Young Adolescents.Journal of Youth and Adolescence, 42(7), 10878-1089.

MacDonald, B. (2012). Using Self-Assessment to Support Individualized Learning.Mathematics Teaching, 231, 26-27.

Navarro, J.J., Campos, M.E, y Uceda, X. (2014). Investigación acción con estudiantes de integración social. Una experiencia basada en la relación educativa. Contextos Educativos: Revista de Educación, 17, 91-108.

Newberry, M. (2013).Reconsidering Differential Behaviors: Reflection and Teacher Judgment When Forming Classroom Relationships. Teacher Development, 17(2), 195-213.

Patton, M. Q. (2002). Qualitative Research and Evaluation Methods. Thousand Oaks, CA: Sage.

Picón, E. (2012). Promoting Learner Autonomy through Teacher-Student Partnership Assessment in an American High School: A Cycle of Action Research. PROFILE: Issues in Teachers' Professional Development, 14(2), 145-162.

Powell, L., y Robson, F. (2014). Learner-Generated Podcasts: A Useful Approach to Assessment? Innovations in Education and Teaching International, 51(3), 12-21.

Saldaña, J. (2009). The Coding Manual for Qualitative Researchers.Thousand Oaks, CA: Sage. Sosa, M.J., López, C., y Díaz, S. (2014). Comunidad de aprendizaje y participación social en un curso MOOC. International journal of EducationalResearch and Innovation, 1, 113.

Smith, J. A. y Osborne, M. (2003). Interpretative Phenomenological Analysis. En J.A. Smith (Ed.),Qualitative psychology: A practical guide to research methods. (pp. 53-79). London: Sage.

Strauss, A. \&Corbin, J. (2002). Bases de la investigación cualitativa: Técnicas y procedimientos para desarrollar la teoría fundamentada. Colombia: Universidad de Antioquia.

Van Looy, L.y Goegebeur, W. (2007). Teachers and teacher trainees as classroom researchers: beyond Utopia? Educational Action Research, 15(1), 107-126.

Vavpotic, D., Zvanut, B., yTrobec, I.(2013). A Comparative Evaluation of E-Learning and Traditional Pedagogical Process Elements. Educational Technology y Society, 16(3), 7687.

Varbelow, S. (2014).Instruction, Curriculum and Society: Iterations Based on the Ideas of William Doll. Online Submission, International Journal of Instruction, 5(1), 87-98.

Vaughan, M. (2014). Flipping the Learning: An Investigation into the Use of the Flipped Classroom Model in an Introductory Teaching Course.Education Research and Perspectives, 14(1), 25-41.

Vrieling, E., Bastiaens, T., y Stijnen, S. (2012). Consequences of Increased Self-Regulated Learning Opportunities on Student Teachers' Motivation and Use of Metacognitive Skills.Australian Journal of Teacher Education, 37(8), 12-23. 